\title{
Application Study of Continuous Nursing Intervention among Ischemic Stroke Patients
}

\author{
Shi-Yun Tan ${ }^{1}$, Yan-Mei Liu, ${ }^{2, *}$ Jia Yang ${ }^{2}$, Meng-Li Chen ${ }^{2}$, Yun-Jie Tong ${ }^{1}$ \\ ${ }^{1}$ Shaanxi University of Chinese Medicine, Xianyang, China. \\ ${ }^{2}$ Shaanxi Traditional Chinese Medicine Hospital, Xi’ an, Shaanxi, China.
}

How to cite this paper: Shi-Yun Tan, Yan-Mei Liu, Jia Yang, Meng-Li Chen, Yun-Jie Tong. (2020) Application Study of Continuous Nursing Intervention among Ischemic Stroke Patients. International Journal of Clinical and Experimental Medicine Research, 4(3), 107-111. DOI: 10.26855/ijcemr.2020.07.013

Received: June 20, 2020

Accepted: July 17, 2020

Published: July 29, 2020

*Corresponding author: Yan-Mei Liu, Shaanxi Traditional Chinese Medicine Hospital, Xi' an, Shaanxi, China.

Email: 1305999760@qq.com

\begin{abstract}
Objective: To analyze the effect of continuous nursing on the prognosis of patients with Ischemic stroke. Methods: A total of 100 patients with Ischemic stroke diagnosed and discharged in Shaanxi Provincial Hospital of Traditional Chinese Medicine from December 2018 to May 2019 were divided into control group and observation group, 50 cases each. The control group received routine nursing, and the observation group received continuous nursing. Treatment compliance, quality of life scores, self-Rating depression scale (SDS), self-rating depression scale (SAS) and National Institutes of Health Stroke Scale (NIHSS) scores, as well as half-year and one-year mortality rates were analyzed and compared between the two groups. Results: Compared with the control group, the treatment compliance, quality of life scores, SDS, SAS and NIHSS scores of the observation group showed statistically significant differences $(\mathrm{P}<0.05)$. Compared with the control group, the mortality rate of the observation group was always lower than that of the control group, but the difference was not statistically significant. Conclusion: The application of continuous nursing in patients with Ischemic stroke can significantly improve the patient's treatment compliance and improve the quality of life of patients. At the same time, the SDS, SAS and NIHSS scores are also greatly improved, which has a positive effect on the patient's prognosis.
\end{abstract}

\section{Keywords}

Ischemic stroke, continuous care, SAS, SDS, NIHSS

\section{Introduction}

Stroke is a group of cerebrovascular diseases characterized by focal neurological deficits, and the onset is relatively urgent. It has become a global health problem with its high incidence, high recurrence rate, high disability rate and high mortality rate [1]. The World Stroke Organization (WSO) released a report in 2019 globally showing currently more than 80 million stroke patients, and more than 13.7 million new stroke patients occur each year [2]. Stroke can be divided into ischemic stroke and hemorrhagic stroke, among which ischemic stroke accounts for about $80 \%$ of stroke incidence. Due to its high morbidity, mortality and disability rate, it has become one of the serious public health problems in China [3-4].

In China, $80 \%$ of stroke patients have left serious sequelae (such as hemiplegia, joint stiffness, contracture, verbal slurred speech, mental retardation, etc.), and the disability rate is as high as $75 \%$ [5]. The long treatment and rehabilitation process brings certain pressure to patients, which affects the quality of life of patients [6].

Studies have shown that many patients still have health care needs after discharge, and the readmission rate of 
patients 30 days after discharge is as high as $20 \%$ [7-8].

It can be seen that the patient is in urgent need of continuous care after discharge.

The purpose of this study is to provide a reference for the continuous nursing after discharge of stroke patients by exploring the application effect of continuous nursing in patients with ischemic stroke.

\section{Object and methods}

\subsection{Objects of Study}

A total of 100 patients with ischemic stroke treated in Shaanxi Provincial Hospital of Traditional Chinese Medicine from December 2018 to May 2019 were selected and included into the control group and observation group according to the random number table method, 50 patients in each group.

Inclusion criteria were: (1) refer to the diagnostic criteria of the fourth national cerebrovascular disease, and brain magnetic resonance imaging (MRI) or computed tomography (CT) confirmed as ischemic stroke [9]; (2) age of $\geqslant 18$ years; (3) clear consciousness and patients can use language or text communication; (4) Patients volunteered to participate and signed informed consents. Exclusion criteria were: (1) comorbid major diseases, such as malignant tumors; (2) patients with combined mental illness; (3) participate in other research projects at the same time; (4) not cooperate with this research.

This study is approved by the Shaanxi Province Hospital of traditional Chinese medicine Institutional Review Board.

\subsection{Methods}

The control group received routine nursing: On the day the patient was discharged, the nursing staff followed the discharge instructions, informed the patient and explained in detail the dosage and dosage of each drug. Explain the matters needing attention in rehabilitation training, diet and life; Health brochures are distributed to patients instructing them to read them in detail to facilitate their recovery.

Patients in the observation group received continuous care, with specific contents as follows: (1) Establishment of a Continuing Care Unit: A continuous nursing team is formed by the attending physician of our hospital, the nurse in charge, the nursing staff and the relevant nursing staff of the community hospital to which the patient belongs. The work responsibilities of each group member are carefully divided so that each group member can carry out nursing intervention for the patient in accordance with the relevant responsibilities. (2) Develop nursing procedures: 1 to 2 days before the patient is discharged from the hospital, the nurse carried out a comprehensive assessment and examination of the patient, and makes specific measures for continuous care based on the examination results; Establish a WeChat communication platform according to the community, publish the relevant matters needing attention in the recovery period of stroke in the WeChat group every day, and timely answer the doubts of patients and their families in the WeChat group. In the first month after discharge, every 7 days, the second month after discharge, every 2 weeks, from the third month after discharge, every other month, the nursing staff in the community implement family follow-up for patients respectively Interview and feedback to the research team to master the changes of the patient's condition and the specific situation of rehabilitation treatment. (3) Specific content of continuous care: When the patient is followed up at home, the nursing staff should understand the patient's medication, blood pressure, daily life, diet, body movement and other conditions in detail, and explain in detail where the patient has questions, so that the patient can have a good recovery; Encourage and guide patients to carry out rehabilitation treatment in strict accordance with the doctor's orders, so that patients can carry out rehabilitation training in accordance with the corresponding plan; When communicating with the patient, remind the patient of the next visit time; Information on disease prevention and control, rehabilitation exercise, diet, daily life and other related information is released on WeChat platform to improve the rehabilitation effect of patient.

\subsection{Observation indexes, evaluation standards}

\section{Observation indexes}

After 6 months of follow-up, the treatment compliance of patients in both groups was evaluated. After 6 and 12 months of follow-up, the quality of life scores and SDS, SAS and NIHSS scores were evaluated.

\section{Evaluation standards}

Treatment compliance: The self-made rating scale of our hospital was adopted, including self-measurement of blood pressure, taking prescribed medicine, adherence to rehabilitation exercise, reasonable diet and timely fol- 
low-up. Complete compliance: all five of the patients did; Partial compliance: taking medicine as prescribed or reaching 3 or more; Noncompliance: not taking prescribed medicine and not reaching three of the other items.

Quality of Life Score: The Barthel Index was used to evaluate the quality of life of the patients. A high score indicates a better quality of life.

SDS, SAS and NIHSS scores: The self-rating depression scale (SDS), self-rating depression scale (SAS) and National Institutes of Health Stroke Scale (NIHSS) scores, were used to assess the patients' negative emotions and neurological functions. The lower the score, the better the patients' condition recovery [10].

\section{Statistical analysis}

SPSS 25.0 software was used for data analysis and processing. The measurement data were presented as mean \pm standard deviation ( $\bar{X} \pm s$ ), and comparison between groups was carried out using t-test. The count data is expressed as a percentage (\%), and Chi-square test was performed for the comparison between groups. $\mathrm{P}<0.05$ was considered statistically significant.

\section{Results}

\section{Comparison of Basic features of included cases between the two groups}

A total of 100 patients were included, including 50 patients in the observation group and 50 patients in the control group. In the observation group, there were 29 male patients and 21 female patients; in the control group, there were 34 male patients and 16 female patients. The average age of patients in the experimental group was $65.28 \pm 5.35$ years old, while that of patients in the control group was $64.24 \pm 6.26$ years old. The average length of hospital stay was $12.25 \pm 4.58$ days and $14.18 \pm 3.37$ days. All baseline data were tested and the difference was not statistically significant $(\mathrm{P}>0.05)$, indicating comparability.

\section{Comparison of Treatment compliance between the two groups}

After 6 months of intervention, 32 patients in the observation group were completely compliant, 10 were partially compliant, and only 5 were non-compliant. In the control group, 20 patients were completely compliant, 11 were partially compliant, and up to 12 were non-compliant. The difference between groups was statistically significant (P $<0.05$ ), see Table 1 .

Table 1. Treatment compliance score

\begin{tabular}{|c|c|c|c|c|}
\hline Items & observation & group & control & $\mathrm{P}$ \\
\hline Full compliance & 32 & & 20 & $<0.05$ \\
\hline Part of the compliance & 10 & & 11 & $<0.05$ \\
\hline noncompliance & 5 & & 12 & $<0.05$ \\
\hline
\end{tabular}

\section{Comparison of quality of life score comparison between the two groups}

At discharge, there was no statistical significance difference in quality of life scores between the two groups (P > 0.05). After 6 months of intervention, the quality of life scores of patients in the observation group were $76.24 \pm 4.35$, higher than those in the control group, and the difference between the two groups was statistically significant $(\mathrm{P}<0.001)$. After further observation for 1 year, the quality of life scores of patients in the observation group and the control group were $80.26 \pm 5.78$ points and $69.37 \pm 5.33$ points respectively, indicating statistically significant differences between the two groups $(\mathrm{P}<0.001)$, see Table 2 .

Table 2. Comparison of Quality of Life Score between two groups after treatment

\begin{tabular}{|c|c|c|c|c|c|}
\hline Items & $\mathbf{n}$ & observation group & control group & $\mathbf{t}$ & $\mathbf{P}$ \\
\hline $\mathrm{T} 1$ & $50 / 50$ & $34.76 \pm 3.48$ & $35.43 \pm 2.87$ & 1.05 & $>0.05$ \\
\hline $\mathrm{T} 2$ & $47 / 44$ & $76.24 \pm 4.35$ & $58.66 \pm 6.42$ & 16.03 & $<0.001$ \\
\hline Т3 & $43 / 40$ & $80.26 \pm 5.78$ & $69.37 \pm 5.33$ & 9.79 & $<0.001$ \\
\hline
\end{tabular}

\section{Comparison of SDS, SAS and NIHSS scores between the two groups}

At discharge, SDS, SAS and NIHSS scores of the two groups were compared, and the difference between the two groups was not statistically significant $(\mathrm{P}>0.05)$. After half a year of observation, the SDS score of the observation group was $40.06 \pm 4.60$, SAS score was $40.06 \pm 4.60$, NIHSS score was $10.52 \pm 2.24$, respectively lower 
than those in the control group, and the differences were statistically significant $(\mathrm{P}<0.05)$. Further observation showed that one year later, although SDS, SAS and NIHSS scores of patients in each group decreased, the scores of patients in the observation group were significantly lower than those in the control group $(\mathrm{P}<0.05)$, see Table 3.

Table 3. Comparison of SDS, SAS and NIHSS scores between two groups after treatment

\begin{tabular}{|c|c|c|c|c|c|}
\hline Items & $\mathbf{n}$ & observation group & control group & $\mathbf{t}$ & $\mathbf{P}$ \\
\hline \multicolumn{6}{|l|}{ SDS } \\
\hline $\mathrm{T} 1$ & $50 / 50$ & $64.68 \pm 5.21$ & $63.95 \pm 6.09$ & 0.64 & 0.52 \\
\hline $\mathrm{T} 2$ & $47 / 44$ & $40.06 \pm 4.60$ & $48.85 \pm 7.12$ & 7.04 & $<0.001$ \\
\hline T3 & $43 / 40$ & $33.02 \pm 4.79$ & $42.99 \pm 5.45$ & 8.87 & $<0.001$ \\
\hline \multicolumn{6}{|l|}{ SAS } \\
\hline $\mathrm{T} 1$ & $50 / 50$ & $68.24 \pm 3.61$ & $67.91 \pm 5.22$ & 0.37 & 0.71 \\
\hline $\mathrm{T} 2$ & $47 / 44$ & $40.06 \pm 4.60$ & $50.61 \pm 4.38$ & 6.53 & $<0.001$ \\
\hline T3 & $43 / 40$ & $37.13 \pm 4.12$ & $46.33 \pm 6.13$ & 8.08 & $<0.001$ \\
\hline \multicolumn{6}{|l|}{ NIHSS } \\
\hline $\mathrm{T} 1$ & $50 / 50$ & $22.35 \pm 3.65$ & $22.09 \pm 4.47$ & 0.32 & 0.75 \\
\hline $\mathrm{T} 2$ & $47 / 44$ & $10.52 \pm 2.24$ & $13.94 \pm 3.74$ & 5.33 & $<0.001$ \\
\hline Т3 & $43 / 40$ & $8.39 \pm 2.83$ & $10.24 \pm 3.17$ & 2.81 & $<0.001$ \\
\hline
\end{tabular}

\section{Comparison of mortality rate between the two groups}

The mortality rates of the two groups were calculated after half a year and one year respectively. There were 3 deaths in the observation group (6\%) and 6 deaths $(12 \%)$ in the control group. The death rate of the observation group after one year was 7 (14\%) and that of the control group (20\%). There was no significant difference between the two groups $(\mathrm{P}<0.05)$, see Table 4.

Table 4. Comparison of mortality rate between two groups after treatment

\begin{tabular}{|c|c|c|c|}
\hline Items & observation group & control group & $\mathbf{P}$ \\
\hline $\mathrm{T} 2$ & $3(6 \%)$ & $6(12 \%)$ & 0.48 \\
\hline Т3 & 7 (14\%) & $10(20 \%)$ & 0.42 \\
\hline
\end{tabular}

Note: T1 represents before discharge, T2 represents half a year after discharge, T3 represents one year after discharge.

\section{Discussion}

About 5.5 million people die of stroke every year in the world. At present, stroke is the main cause of death in China and has become a heavy disease burden [11]. Stroke is prone to a variety of dysfunction, seriously affecting the physical and mental health of patients. Long term mortality after stroke and related prognostic factors have been studied in various countries. The cumulative mortality rate is $13.6 \%$ to $34.2 \%$ in one year and $29.1 \%$ to $41.7 \%$ in five years [12]. Several major prognostic factors known to be associated with long-term mortality after stroke are stroke age, gender, type of comorbidities, and characteristics of medical care in hospitals. In developed countries, "organized stroke unit care” reduces early post-stroke mortality by providing acute care and preventing medical complications [13]. The purpose of this study was to explore whether continuity of care after discharge can improve the prognosis of patients.

Our study found that compared with the control group, patients in the observation group who were given continuous care had higher treatment compliance after 6 months of follow-up; although the scores of quality of life of the two groups were improved after one year of follow-up, the quality of life score of the observation group was always better than that of the control group; we evaluated the SDS and SAS of the patients in each group at the time of discharge, half a year and one year later The results showed that continuous nursing intervention had a positive effect on the physical and mental recovery of the patients. The SDS, SAS and NIHSS scores of the observation group were lower than those of the control group after half a year and one year. In the evaluation of mortality, the mortality of the observation group was always lower than that of the control group, but the differ- 
ence was not statistically significant. In the actual clinical, no statistical significance does not mean that there is no clinical significance, continuity of care can reduce the number of deaths of patients, has a certain positive effect on patients. Therefore, continuous nursing can affect the behavior and life style of stroke patients, actively deal with negative emotions, improve their treatment compliance, so as to reduce the rehospitalization rate and recurrence rate of stroke patients, and improve their quality of life.

\section{Declaration}

All authors of this article declare they have no conflicts of interest.

\section{Acknowledgements}

This study was approved by the Shaanxi Provincial Department of Science and Technology (2020KRM141) and the Innovation project for graduate students of university-level scientific research projects of Shaanxi University of Traditional Chinese Medicine in 2020 for funding the project.

\section{References}

[1] Roger VL, Go AS, Lloyd-Jones DM, et al. (2012). Heart disease and stroke statistics—2015 update A Report from the American Heart [J]. Circulation, 2012, 125(1): 188-97.

[2] Lindsay MP, Norrving B, Sacco RL, et al. (2019). World Stroke Organization (WSO): Global Stroke Fact Sheet 2019 [J]. International Journal of Stroke, 14(8): 806-817.

[3] Thrift AG, Thayabaranathan, T, Donnan, GA, et al. (2017). Global stroke statistics [J]. International Journal of Stroke, 2017, 9(1): 13-32.

[4] Valery L Feigin, Emma Nichols, Tahiya Alam, et al. (2019). Global, Regional, and National Burden of Neurological Disorders, 1990-2016: a Systematic Analysis for the Global Burden of Disease Study 2016 [J]. The Lancet Neurology, 2019, 18(5): 439-458

[5] Wang LD, Liu JM, Yang Y, et al. (2019). Prevention and treatment of stroke in China still faces great challenges—Summary of Chinese Stroke Prevention and Treatment Report 2018 [J]. Chin J Circulation, 2019, 34(2): 105-119.

[6] Li QY. (2020). Effect of continuous nursing on health behavior, self-care ability and quality of life of stroke patients [J]. Journal of Chronic Epidemiology, 2020, 21(04): 581-582+585.

[7] Jiang ZZ, Zhang YQ. (2020). Influence of continuance nursing based on doda concept on functional rehabilitation and negative emotion of stroke patients [J]. Chinese Journal of Modern Nursing, 2020, 26(15): 2069-2072.

[8] Tan SY, Liu YM, Bai WW. (2020). Application progress of traditional Chinese medicine continuous nursing in patients with hemiplegia and shoulder pain after stroke [J]. Generalnursing, 2020, 18(10): 1197-1199.

[9] The Fourth National Cerebrovascular Disease Conference. (1996). Main points for diagnosis of various cerebrovascular diseases [J]. Chinese Journal of Neurology, 1996, 29(6): 60-61.

[10] Zhang ZJ. (2005). Manual of behavioral Medicine Scales [M]. Beijing: China Medical Electronic Audio-visual Publishing House, 2005: 213-214.

[11] Johnson CO, Minh N, Roth GA, et al. (2019). Global, regional, and national burden of stroke, 1990-2016: a systematic analysis for the Global Burden of Disease Study 2016 [J]. LANCET Neurology, 2019, 18(5): 439-458.

[12] Cruz-Cruz C, Moreno-Macias H, Del Socorro Parra-Cabrera M, et al. (2019). Survival after Ischemic and Hemorrhagic Stroke: A 4-Year Follow-Up at a Mexican Hospital [J]. Journal of Stroke \& Cerebrovascular Diseases, 2019, 28(8): 2109-2114.

[13] Towfighi A, Saver JL. (2011). Stroke Declines From Third to Fourth Leading Cause of Death in the United States Historical Perspective and Challenges Ahead [J]. STROKE, 2011, 42(8): 2351-2355. 